\title{
EXTENSION OF EMBEDDINGS CLOSE TO ISOMETRIES OR SIMILARITIES
}

\author{
P. TUKIA and J. VÄISÄLÄ
}

\section{Introduction}

Let $X$ be a subset of the euclidean $n$-space $R^{n}$. A map $f: X \rightarrow R^{n}$ is said to be bilipschitz if there is $L \geqq 1$ such that

$$
|x-y| / L \leqq|f(x)-f(y)| \leqq L|x-y|
$$

for all $x, y \in X$; we also say that $f$ is $L$-bilipschitz. It is not usually possible to extend $f$ to a bilipschitz homeomorphism $g: R^{n} \rightarrow R^{n}$. For example, if $X$ is the circle $S^{1}$ and if $n=3, f X$ can be knotted, and $f$ cannot be extended to any homeomorphism of $R^{3}$. Or if $X=S^{2}$ and $n=3, f X$ can be the Fox-Artin wild sphere [ $\mathrm{Ge}_{1}$, Theorem 3]. On the other hand, if $X=S^{1}$ and $n=2$, the extension is possible [ $\mathrm{Tu}_{1}, \mathrm{Tu}_{2}, \mathrm{JK}$, La]. More generally, $X$ can be a quasicircle in $R^{2}\left[\mathrm{Ge}_{2}\right.$, Corollary 2, p. 218].

In this paper we show that the extension is possible in the case where $X$ is $R^{p}$ or $S^{p}, 1 \leqq p \leqq n-1$, and $L$ is sufficiently close to 1 . Moreover, we show that the extension $g: R^{n} \rightarrow R^{n}$ can be chosen to be $L_{1}$-bilipschitz where $L_{1}=L_{1}(L, n)$ and $\lim _{L \rightarrow 1} L_{1}(L, n)=1$. For example, for sufficiently small $L$, an $L$-bilipschitz image of $S^{1}$ in $R^{3}$ is unknotted.

Our method is rather elementary and explicit, especially in the case $p=n-1$. If $X=R^{p}$, we choose a suitable triangulation on $R^{n} \backslash X$, define $g$ at the vertices and extend $g$ affinely to the simplexes. Thus $g$ will be PL outside $X$. The case $X=S^{p}$ reduces to the case $X=R^{p}$ by means of an auxiliary inversion.

Corresponding results are also proved for quasisymmetric embeddings with small dilatation. The definition and some basic properties of these maps are recalled in Section 2.

The main results are given in Section 5. Sections 3 and 4 contain auxiliary material.

In a later paper, the second author will consider this extension problem for more general sets $X$. For example, the extension is possible if $X$ is a compact $(n-1)$ dimensional DIFF or PL manifold in $R^{n}$. The basic idea will be the same, but the construction of $g$ will be somewhat less explicit than in the present paper.

We thank Jouni Luukkainen for careful reading of our manuscript and for several valuable suggestions.

doi:10.5186/aasfm.1984.0914 


\section{Preliminaries}

2.1. Notation and terminology. We let $R^{n}$ denote the euclidean $n$-space, $\left(e_{1}, \ldots\right.$ $\left.\ldots, e_{n}\right)$ its standard basis, and $|x|=\left(x_{1}^{2}+\ldots+x_{n}^{2}\right)^{1 / 2}$ the euclidean norm of a vector $x \in R^{n}$. We shall regard $R^{n}$ in the natural way as a subspace of the space $R^{\infty}$ of all sequences of real numbers with only finitely many non-zero terms. In particular, if $p<n, R^{p}$ will be identified with the subspace of $R^{n}$ spanned by $e_{1}, \ldots, e_{p}$. The norm in $R^{\infty}$ is defined as $|x|=\left(x_{1}^{2}+x_{2}^{2}+\ldots\right)^{1 / 2}$.

We shall use the following notation for certain subsets of $R^{n}$ :

$$
\begin{gathered}
R_{+}^{n}=\left\{x \in R^{n}: x_{n} \geqq 0\right\}, \\
I^{n}=[-1,1]^{n}, \\
J^{n}=[0,1]^{n}, \\
B^{n}(x, r)=\left\{y \in R^{n}:|y-x|<r\right\}, \\
S^{n-1}(x, r)=\partial B^{n}(x, r)=\left\{y \in R^{n}:|y-x|=r\right\}, \\
B^{n}(r)=B^{n}(0, r), B^{n}=B^{n}(1), \\
S^{n-1}(r)=S^{n-1}(0, r), S^{n-1}=S^{n-1}(1) .
\end{gathered}
$$

If $A \subset R^{n}$, we let $T(A)$ denote the affine subspace spanned by $A$. If $f$ and $g$ are two maps into $R^{n}$, defined in a set $A$, we set

$$
\|f-g\|_{A}=\sup _{x \in A}|f(x)-g(x)| .
$$

2.2. Bilipschitz maps. Let $X$ and $Y$ be metric spaces. The distance between points $a, b$ in either space is written as $|a-b|$. As defined in the introduction, a map $f: X \rightarrow Y$ is $L$-bilipschitz if it satisfies the double inequality (1.1). If the right-hand inequality of (1.1) is satisfied, $f$ is said to be L-Lipschitz. The smallest such $L$ is then called the Lipschitz constant of $f$ and denoted by $\operatorname{lip} f$. A 1-bilipschitz map is an isometry. If $A \subset R^{n}$, an isometry $f: A \rightarrow R^{n}$ is always the restriction of a unique affine isometry $f_{1}: T(A) \rightarrow R^{n}$. If $E$ is a vector subspace of $R^{n}$ and if $f: E \rightarrow R^{n}$ is an isometry with $f(0)=0, f$ is an orthogonal map. A sense-preserving orthogonal map $f: R^{n} \rightarrow R^{n}$ is called a rotation.

2.3. Quasisymmetric maps. These maps were introduced in [TV]. We recall the definition. Let $X$ and $Y$ be metric spaces. An embedding $f: X \rightarrow Y$ is quasisymmetric (abbreviated QS) if there is a homeomorphism $\eta: R_{+}^{1} \rightarrow R_{+}^{1}$ such that if $a, b, x \in X$ with $|a-x| \leqq t|b-x|$, then $|f(a)-f(x)| \leqq \eta(t)|f(b)-f(x)|$. We also say that $f$ is $\eta$-QS. An $L$-bilipschitz map is $\eta$-QS with $\eta(t)=L^{2} t$. If $f$ is $\eta-\mathrm{QS}$ with $\eta=\mathrm{id}$, $f$ is said to be a similarity. Then there is $L>0$ such that $|f(x)-f(y)|=L|x-y|$ for all $x, y \in X$. A map $f: R^{p} \rightarrow R^{n}$ is a similarity if and only if it is of the form $f(x)=$ $\lambda A(x)+b$, where $\lambda>0, b \in R^{n}$ and $A: R^{p} \rightarrow R^{n}$ is an orthogonal map. 
We shall consider $\eta-$ QS maps which are close to similarities, that is, $\eta$ is close to the identity map of $R_{+}^{1}$. We need a measure for this closeness. To this end, observe that the sets

$$
N(\mathrm{id}, s)=\{\eta:|\eta(t)-t| \leqq s \text { for } 0 \leqq t \leqq 1 / s\},
$$

$s>0$, form a basis for the neighborhood system of id in the compact-open topology of the space $H\left(R_{+}^{1}\right)$ of all homeomorphisms $\eta: R_{+}^{1} \rightarrow R_{+}^{1}$. We say, somewhat ambiguously, that an embedding $f: X \rightarrow Y$ is $s$-quasisymmetric if it is $\eta-\mathrm{QS}$ for some $\eta \in N(\mathrm{id}, s)$. We also say that $f$ is $0-\mathrm{QS}$ if it is a similarity.

Every $L$-bilipschitz map is $s-\mathrm{QS}$ with $s=\left(L^{2}-1\right)^{1 / 2}$. For connected spaces we have the following useful criterion:

2.4. Lemma. Let $X$ and $Y$ be metric spaces with $X$ connected. Let $0<s \leqq 1 / 6$, and let $f: X \rightarrow Y$ be an embedding with the following property: If $a, b, x \in X$ are distinct points with $|a-x| /|b-x|=t \in[s, 1 / s]$, then

$$
\frac{|f(a)-f(x)|}{|f(b)-f(x)|}=t^{\prime} \leqq t+s .
$$

Then $f$ is $\eta_{s}-\mathrm{QS}$ with some $\eta_{s}$ depending only on $s$, and $f$ is $2 s-\mathrm{QS}$.

Proof. We first show that if $t<s$, then $t^{\prime} \leqq 2 s$. Choose an integer $m \geqq 0$ such that $s^{m+2} \leqq t<s^{m+1}$. Since $X$ is connected, we can find points $x_{0}, \ldots, x_{m+1}$ in $X$ such that

$$
\begin{gathered}
x_{0}=b, \\
\left|x_{j+1}-x\right|=s\left|x_{j}-x\right| \text { for } 0 \leqq j \leqq m-1, \\
\frac{\left|x_{m+1}-x\right|}{\left|x_{m}-x\right|}=\frac{|a-x|}{\left|x_{m+1}-x\right|}=s^{\prime}
\end{gathered}
$$

where $s \leqq s^{\prime}=\left(t / s^{m}\right)^{1 / 2}<s^{1 / 2}$. Then

for $0 \leqq j \leqq m-1$, and

$$
\frac{\left|f\left(x_{j+1}\right)-f(x)\right|}{\left|f\left(x_{j}\right)-f(x)\right|} \leqq 2 s<1
$$

because $s \leqq 1 / 6$.

$$
t^{\prime}=\frac{|f(a)-f(x)|}{|f(b)-f(x)|} \leqq\left(s^{\prime}+s\right)^{2}<\left(s^{1 / 2}+s\right)^{2} \leqq 2 s,
$$

The first assertion of the lemma follows now from [TV, 3.10] with the substitution $\lambda_{1}=\lambda_{2}=1 / 2, h=2, H=s+1 / s$. The second assertion follows from the first one and from what was proved above.

2.5. Quasisymmetry is closely related to quasiconformality. In particular, if $G$ is open in $R^{n}$, an $\eta$-QS embedding $f: G \rightarrow R^{n}$ is $K$-quasiconformal with $K=\eta(1)^{n-1}$ [Vä, 2.3$]$. Conversely, a quasiconformal embedding $f: G \rightarrow R^{n}$ need not be QS, 
but this is true if $G=R^{n}\left[\mathrm{Vä}_{2}, 2.5\right]$. In this case, the closeness of $f$ to a similarity can also be expressed in terms of the dilatation $K$ :

2.6. Theorem. Let $f: R^{n} \rightarrow R^{n}$ be an embedding, $n \geqq 2$. If $f$ is $s-\mathrm{QS}, f$ is $K^{-}$ quasiconformal where $K=K(s, n) \rightarrow 1$ as $s \rightarrow 0$. Conversely, if $f$ is $K$-quasiconformal, $f$ is $s-$ QS where $s=s(K, n) \rightarrow 0$ as $K \rightarrow 1$. Moreover $f R^{n}=R^{n}$.

Proof. For $s \leqq 1$, we already gave the first part of the theorem in 2.5 with $K(s, n)=(1+s)^{n-1}$. We omit the proof of the case $s \geqq 1$, since it is not needed in this paper.

Suppose that the second part is false. Then there are $s \in(0,1 / 6]$ and a sequence of $K_{j}$-quasiconformal maps $f_{j}: R^{n} \rightarrow R^{n}$ such that $K_{j} \rightarrow 1$ and no $f_{j}$ is $2 s-\mathrm{QS}$. By 2.4, we can pick points $a_{j}, b_{j}, x_{j} \in R^{n}$ such that

$$
\frac{\left|a_{j}-x_{j}\right|}{\left|b_{j}-x_{j}\right|}=t_{j} \in[s, 1 / s], \quad \frac{\left|f_{j}\left(a_{j}\right)-f_{j}\left(x_{j}\right)\right|}{\left|f_{j}\left(b_{j}\right)-f_{j}\left(x_{j}\right)\right|}=t_{j}^{\prime} \geqq t_{j}+s .
$$

Performing auxiliary similarities, we may assume that $x_{j}=0=f_{j}\left(x_{j}\right)$ and $a_{j}=e_{1}=f_{j}\left(a_{j}\right)$. Since $\left|b_{j}\right|=1 / t_{j} \in[s, 1 / s]$, we may assume that $b_{j} \rightarrow b \in R^{n} \backslash\{0\}$. Furthermore, we may assume that the maps $f_{j}$ converge to an isometry $f: R^{n} \rightarrow R^{n}$ uniformly on compact sets. Then

$$
\lim _{j \rightarrow \infty} t_{j}=\frac{1}{|b|}=\frac{1}{|f(b)|}=\lim _{j \rightarrow \infty} t_{j}^{\prime} \geqq \lim _{j \rightarrow \infty} t_{j}+s,
$$

which is a contradiction.

The final statement is a well-known property of quasiconformal maps [V $\ddot{a}_{1}$, 17.4].

\section{Elementary estimates}

3.1. In this section we give some elementary inequalities for affine maps in $R^{n}$ and consider the approximation of QS maps by similarities. We first introduce some notation. Let $\Delta=a_{0} \ldots a_{k}$ be a $k$-simplex in $R^{n}$ with vertices $a_{0}, \ldots, a_{k}$. We let $b_{j}$ denote the distance of $a_{j}$ from the $(k-1)$-plane spanned by the opposite face, and we set $b(\Delta)=\min \left(b_{0}, \ldots, b_{k}\right)$. The diameter $d(\Delta)$ of $\Delta$ is the largest edge $\left|a_{i}-a_{j}\right|$. We let $\Delta^{0}$ denote the set of the vertices of $\Delta$.

The following lemma is obvious:

3.2. Lemma. Let $\beta_{j}: \Delta \rightarrow[0,1]$ be the $j$-th barycentric coordinate. Then $\beta_{j}$ is $b_{j}^{-1}$-Lipschitz.

3.3. Theorem. Suppose that $\Delta \subset R^{n}$ is an n-simplex, that $f: R^{n} \rightarrow R^{n}$ is affine, and that $h: R^{n} \rightarrow R^{n}$ is a sense-preserving isometry such that

$$
|h(v)-f(v)| \leqq \alpha b(\Delta) /(n+1)
$$


for every vertex $v$ of $\Delta$, where $0 \leqq \alpha \leqq 1 / 2$. Then $f$ is sense-preserving and $(1+2 \alpha)$ bilipschitz.

Proof. Replacing $f$ by $h^{-1} f$ we may assume that $h=\mathrm{id}$. Let $x, y$ be points in $\Delta$ with barycentric representations

$$
x=\sum_{i=0}^{n} \xi_{i} a_{i}, \quad y=\sum_{i=0}^{n} \eta_{i} a_{i} .
$$

By 3.2 we obtain

$$
|f(x)-f(y)| \leqq|x-y|+\Sigma_{i}\left|\xi_{i}-\eta_{i}\right|\left|f\left(a_{i}\right)-a_{i}\right| \leqq(1+\alpha)|x-y| .
$$

Similarly we obtain the estimate

$$
|f(x)-f(y)| \geqq(1-\alpha)|x-y| .
$$

Since $0 \leqq \alpha \leqq 1 / 2,1-\alpha \geqq(1+2 \alpha)^{-1}$, which implies that $f$ is $(1+2 \alpha)$-bilipschitz.

Define the segmental homotopy $h_{t}: R^{n} \rightarrow R^{n}$ by $h_{t}(x)=(1-t) f(x)+t x$. Then each $h_{t}$ is an affine map satisfying the same conditions as $f$. Thus every $h_{t}$ is $(1+2 a)$ bilipschitz and hence bijective. Therefore $\left(h_{t}\right)$ is an isotopy, which proves that $f$ is sense-preserving.

3.4. To formulate the corresponding result for similarities we use the following notation: If $f: R^{n} \rightarrow R^{n}$ is an affine bijection, we set

$$
L_{f}=\operatorname{lip} f, \quad l_{f}=\left(\operatorname{lip} f^{-1}\right)^{-1}=\min _{|x|=1}|f(x)|, \quad H_{f}=L_{f} / l_{f} .
$$

3.5. Theorem. Suppose that $\Delta \subset R^{n}$ is an n-simplex, that $f: R^{n} \rightarrow R^{n}$ is affine, and that $h: R^{n} \rightarrow R^{n}$ is a sense-preserving similarity such that

$$
|h(v)-f(v)| \leqq \alpha L_{h} b(\Delta) /(n+1)
$$

for every vertex $v$ of $\Delta$, where $0 \leqq \alpha \leqq 1 / 2$. Then $f$ is sense-preserving, and

$$
L_{f} \leqq L_{h}(1+2 \alpha), \quad l_{f} \geqq L_{h} /(1+2 \alpha), H_{f} \leqq(1+2 \alpha)^{2} .
$$

Proof. Apply 3.3 with the substitution $f \mapsto f / L_{h}, h \mapsto h / L_{h}$.

We omit the elementary but somewhat tedious proof of the following obvious fact:

3.6. Lemma. Let $K$ be a finite simplicial complex in $R^{n}$, and let $K^{0}$ be the set of vertices of $K$. Then there is a positive number $r_{0}=r_{0}(K)$ such that if $f:|K| \rightarrow R^{n}$ is affine in each simplex of $K$ and if $h$ is a similarity such that $\|f-h\|_{K^{0}} \leqq L_{h} r_{0}$, then $f$ is injective. If $u$ is a similarity, $r_{0}(u K)=L_{u} r_{0}(K)$.

The following elementary result is well-known:

3.7. Lemma. Let $\Delta=a_{0} \ldots a_{k}$ be a $k$-simplex in $R^{n}$, let $r_{j}>0$ for $0 \leqq j \leqq k$, and let

$$
S=\left\{x \in R^{n}:\left|x-a_{j}\right|=r_{j} \text { for } 0 \leqq j \leqq k\right\} .
$$


Then there are three possibilities:

(1) $S=\emptyset$.

(2) $S$ consists of a single point in $T(\Delta)$.

(3) $S$ is a sphere of dimension $n-k-1$ with center in $T(\Delta)$ and such that $T(S)$ is perpendicular to $T(\Delta)$.

3.8. Remark. Consider the case $k=n-1$ in 3.7. Then in the case (3), $S$ consists of two points which are symmetric with respect to $T(\Delta)$. Consequently, if $\Delta$ is an $n$-simplex with given $n$ vertices $a_{0}, \ldots, a_{n-1}$ and given edges $\left|a_{n}-a_{j}\right|$, there are exactly two possibilities for $a_{n}$. If the orientation of $\Delta$ is given, there is only one possibility.

It follows that a sense-preserving isometry $h: R^{n} \rightarrow R^{n}$ is uniquely determined by $h \mid \sigma$ for any $(n-1)$-simplex $\sigma$.

3.9. Lemma. Let $\Delta=a_{0} \ldots a_{n-1}$ be an $(n-1)$-simplex in $R^{n}, n \geqq 2$, and let $x \in R^{n}$ with $|x| \leqq r$. Let $f: \Delta^{0} \cup\{x\} \rightarrow R^{n}$ be an $s-\mathrm{QS}$ map which in the case $x \notin T(\Delta)$ does not change the orientation. If $\left|f\left(a_{j}\right)-a_{j}\right| \leqq \delta$ for $0 \leqq j \leqq n-1$, then

$$
|f(x)-x| \leqq \varepsilon(s, \delta, r, \Delta)
$$

where $\varepsilon(s, \delta, r, \Delta) \rightarrow 0$ as $s \rightarrow 0$ and $\delta \rightarrow 0$.

Proof. Suppose that the lemma is false. Then there are sequences $s_{k} \rightarrow 0$ and $\delta_{k} \rightarrow 0$ and a sequence of $s_{k}$-QS maps $f_{k}: \Delta^{0} \cup\{x\} \rightarrow R^{n}$ satisfying the condition on orientation such that $\left|f_{k}\left(a_{j}\right)-a_{j}\right| \leqq \delta_{k}$ for $0 \leqq j \leqq n-1$ and $\left|f_{k}(x)-x\right| \geqq \varepsilon$ for some $\varepsilon>0$ and for all $k$. Passing to a subsequence, we may assume that $f_{k}(x) \rightarrow y \in R^{n}$. Since $f_{k}\left(a_{j}\right) \rightarrow a_{j}$ for all $j, f_{k}$ converges to a map $f$. Since $s_{k} \rightarrow 0, f$ is a similarity. Since $f \mid \Delta^{0}=\mathrm{id}, f$ is an isometry. If $x \in T(\Delta), x=y$ by 3.7. If $x \notin T(\Delta)$, then $f$ does not change the orientation of the $n$-simplex $a_{0} \ldots a_{n-1} x$, whence $x=y$ by 3.8 . This contradicts the inequality $|f(x)-x| \geqq \varepsilon$.

3.10. Lemma. Suppose that $p \geqq 1$, that $f: R^{p} \rightarrow R^{\infty}$ is $s-\mathrm{QS}$ and that $f(0)=0$, $f\left(e_{j}\right) \in R_{+}^{j}$ for $1 \leqq j \leqq p$. Let $r>0$. If $x \in \bar{B}^{p}(r)$, then

$$
|f(x)-| f\left(e_{1}\right)|x| \leqq \varepsilon(s, p, r)\left|f\left(e_{1}\right)\right|,
$$

where $\lim _{s \rightarrow 0} \varepsilon(s, p, r)=0$.

Proof. Suppose that the lemma is false for some $p$ and $r$. Then there are $\varepsilon>0$ and a sequence of $s_{k}$-QS maps $f_{k}: R^{p} \rightarrow R^{\infty}$ such that $s_{k} \rightarrow 0, f_{k}(0)=0, f_{k}\left(e_{j}\right) \in R_{+}^{j}$ for $1 \leqq j \leqq p$, and such that $\left|f_{k}\left(x_{k}\right)-\right| f_{k}\left(e_{1}\right)\left|x_{k}\right| \geqq \varepsilon\left|f_{k}\left(e_{1}\right)\right|$ for some $x_{k} \in \bar{B}^{p}(r)$. Replacing $f_{k}$ by $f_{k} /\left|f_{k}\left(e_{1}\right)\right|$ we may assume that $f_{k}\left(e_{1}\right)=e_{1}$. We may also assume that $x_{k} \rightarrow x \in \bar{B}^{p}(r)$. Replacing $f_{k}$ by $\psi f_{k}$ where $\psi$ is a suitable isometry of $R^{\infty}$ with $\psi \mid R^{p}=\mathrm{id}$, we may assume that $f_{k}(x) \in R^{p+1}$. Since $s_{k} \rightarrow 0$, it follows by induction from 3.9 that $f_{k}\left(e_{j}\right) \rightarrow e_{j}$ for $j \in[1, p]$ and that $f_{k}(x) \rightarrow x$. On the other hand, the family $\left\{f_{k}: k \in N\right\}$ is equicontinuous by 2.4 and by [TV, 3.4]. Hence $\left|f_{k}\left(x_{k}\right)-f_{k}(x)\right| \rightarrow 0$, which gives the contradiction $\varepsilon \leqq\left|f_{k}\left(x_{k}\right)-x_{k}\right| \rightarrow 0$. 
3.11. Lemma. For every integer $p \geqq 1$ there is $s_{2}=s_{2}(p)>0$ with the following property: If $f$ is an $s_{2}-\mathrm{QS}$ map of the set $E=\left\{0, e_{1}, \ldots, e_{p}\right\}$ into $R^{\infty}$, then the vectors $f\left(e_{j}\right)-f(0), 1 \leqq j \leqq p$, are linearly independent.

Proof. By an auxiliary similarity of $R^{\infty}$, we may assume that $f(0)=0$, that $f\left(e_{1}\right)=e_{1}$, and that $f\left(e_{j}\right) \in R^{p}$ for all $j$. If the lemma is not true, there is a sequence of $s_{k}-$ QS maps $f_{k}: E \rightarrow R^{p}$ satisfying these conditions such that $s_{k} \rightarrow 0$ and such that the vectors $f_{k}\left(e_{j}\right), 1 \leqq j \leqq p$, are linearly dependent. An easy compactness argument leads to a contradiction.

\section{Frames}

4.1. In this section we give auxiliary results on frames in $R^{n}$, which are needed in the case $p \leqq n-2$ of the main theorems in Section 5. For $1 \leqq p \leqq n$, a $p$-frame in $R^{n}$ is a $p$-tuple $v=\left(v^{1}, \ldots, v^{p}\right)$ of linearly independent vectors $v^{j} \in R^{n}$. We let $V_{p}\left(R^{n}\right)$ denote the set of all $p$-frames in $R^{n}$ and $V_{p}^{0}\left(R^{n}\right)$ the subset of all orthonormal $p$-frames. We identify $V_{p}\left(R^{n}\right)$ with an open subset of $\left(R^{n}\right)^{p}=R^{p n}$. However, it is convenient to use the norm

$$
\|v\|=\max \left\{\left|v^{j}\right|: 1 \leqq j \leqq p\right\}
$$

in $\left(R^{n}\right)^{p}$ instead of the euclidean norm $|v|$ of $R^{p n}$. These two norms are equivalent: $\|v\|^{2} \leqq|v|^{2} \leqq p\|v\|^{2}$.

Since $V_{p}^{0}\left(R^{n}\right)$ is compact, we can choose a compact neighborhood $N_{p}^{n}$ of $V_{p}^{0}\left(R^{n}\right)$, contained in $V_{p}\left(R^{n}\right)$. The Gram-Schmidt process defines a retraction $G: V_{p}\left(R^{n}\right) \rightarrow$ $V_{p}^{0}\left(R^{n}\right)$. We shall make use of the fact that $G$ is continuously differentiable (in fact, real analytic). This implies that if $v \in N_{p}^{n}$ and $v+z \in V_{p}\left(R^{n}\right)$ with $\|z\| \leqq q$, we can write

$$
G(v+z)=G(v)+G^{\prime}(v) z+\|z\| \varepsilon(z, v),
$$

where $G^{\prime}(v): R^{p n} \rightarrow R^{p n}$ is a linear map and $\|\varepsilon(z, v)\| \leqq \varepsilon_{n}(q)$ with $\lim _{q \rightarrow 0} \varepsilon_{n}(q)=0$. Moreover,

$$
\left\|G^{\prime}(v)\right\| \leqq M=M(n)
$$

for all $v \in N_{p}^{n}$.

We first prove an interpolation lemma, for which we introduce the following notation: Let $J^{p}$ be the $p$-cube $[0,1]^{p}$. We let $\Gamma_{p}$ denote the family of the $3^{p}$ closed subcubes of $J^{p}$, obtained by trisecting the sides of $J^{p}$. Let $\Gamma_{p}^{\prime}$ denote the subfamily of this "Rubik $p$-cube", consisting of the $2^{p}$ cubes containing the vertices of $J^{p}$. If $\alpha, \beta \in \Gamma_{p}$ with $\alpha \cap \beta \neq \emptyset$, we write $\alpha \sim \beta$.

4.4. Lemma. For every integer $n \geqq 2$ there is a number $q_{0}=q_{0}(n)>0$ with the following property: Let $1 \leqq p \leqq n-1$ and $0 \leqq q \leqq q_{0}$, and let $u: \Gamma_{p}^{\prime} \rightarrow V_{n}^{0}\left(R^{n}\right), v: \Gamma_{p} \rightarrow$ $V_{p}^{0}\left(R^{n}\right)$ be maps, denoted by $\alpha \mapsto u_{\alpha}, \alpha \mapsto v_{\alpha}$, and satisfying the conditions: 
(a) $u_{\alpha}^{j}=v_{\alpha}^{j}$ for $\alpha \in \Gamma_{p}^{\prime}$ and $1 \leqq j \leqq p$.

(b) $\left\|u_{\alpha}-u_{\beta}\right\| \leqq q$ for all $\alpha, \beta \in \Gamma_{p}^{\prime}$.

(c) $\left\|v_{\alpha}-v_{\beta}\right\| \leqq q^{2}$ whenever $\alpha \sim \beta$.

Then there is a map $w: \Gamma_{p} \rightarrow V_{n}^{0}\left(R^{n}\right)$ such that:

(1) $w_{\alpha}^{j}=v_{\alpha}^{j}$ for $\alpha \in \Gamma_{p}$ and $1 \leqq j \leqq p$.

(2) $w \mid \Gamma_{p}^{\prime}=u$.

(3) $\left\|w_{\alpha}-w_{\beta}\right\| \leqq\left(1-2^{-p-1}\right) q$ whenever $\alpha \sim \beta$.

(4) If $\alpha \in \Gamma_{p}$ meets a $k$-dimensional face $A$ of $J^{p}, 0 \leqq k \leqq p$, then $w_{\alpha}$ depends only on $v_{\alpha}$ and on the frames $u_{\beta}$ for which $\beta$ meets $A$.

Proof. Observe that the maps form the diagram

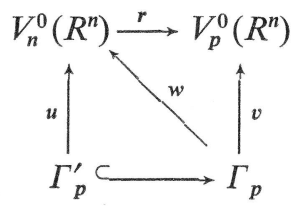

Here $r$ is the natural map $r(v)=\left(v^{1}, \ldots, v^{p}\right)$. The condition (a) means that the rectangle is commutative; the conditions (1) and (2) mean that the two triangles are commutative.

Fix an integer $p \in[1, n-1]$. It suffices to find a number $q_{0}>0$ satisfying the condition of the lemma for this $p$. Let $0 \leqq q \leqq 1$, and let $u, v$ be as in the lemma. In the course of the proof we shall put more restrictions on $q$.

Let $\alpha \in \Gamma_{p}$, and let $A_{\alpha}$ be the face of $J^{p}$ of minimal dimension meeting $\alpha$. Let $B_{\alpha}$ be the family of all $\beta \in \Gamma_{p}^{\prime}$ meeting $A_{\alpha}$. Define $x_{\alpha} \in\left(R^{n}\right)^{n}$ as follows: For $j \leqq p$ set $x_{\alpha}^{j}=v_{\alpha}^{j}$. For $p+1 \leqq j \leqq n$ let $x_{\alpha}^{j}$ be the arithmetic mean of the vectors $u_{\beta}^{j}$ over all $\beta \in B_{\alpha}$. From the conditions (a), (b), (c) it follows that $\left\|x_{\alpha}-u_{\beta}\right\| \leqq q$ for every $\beta \in B_{\alpha}$. Since $N_{n}^{n}$ is a neighborhood of $V_{n}^{0}\left(R^{n}\right)$ in $\left(R^{n}\right)^{n}$, there is $q_{1}=q_{1}(n)>0$ such that $q \leqq q_{1}$ implies $x_{\alpha} \in N_{n}^{n}$. From now on, we assume $q \leqq q_{1}$. We claim that the map $w: \Gamma_{p} \rightarrow V_{n}^{0}\left(R^{n}\right)$, defined by $w_{\alpha}=G\left(x_{\alpha}\right)$, is the desired map, provided that $q$ is sufficiently small. The conditions (1), (2) and (4) are clearly satisfied.

It remains to verify (3). Let $\alpha \in \Gamma_{p}$. There is a positive integer $m(\alpha)$ such that $m(\alpha)$ card $B_{\alpha}=2^{p}$. Set $C_{\alpha}=B_{\alpha} \times\{1, \ldots, m(\alpha)\}$ and define $\bar{u}: C_{\alpha} \rightarrow V_{n}^{0}\left(R^{n}\right)$ by $\bar{u}_{(\beta, k)}=u_{\beta}$. Then

$$
x_{\alpha}^{j}=2^{-p} \sum_{\gamma \in C_{\alpha}} \bar{u}_{\gamma}^{j}
$$

for $p+1 \leqq j \leqq n$.

For $\gamma \in C_{\alpha}$ write

$$
z_{\gamma}=\bar{u}_{\gamma}-x_{\alpha}, \quad z=2^{-p} \sum_{\gamma \in C_{\alpha}} z_{\gamma} .
$$

If $1 \leqq j \leqq p$, (a) and (c) imply

$$
\left|z_{\gamma}^{j}\right|=\left|\bar{u}_{\gamma}^{j}-v_{\alpha}^{j}\right| \leqq q^{2}, \quad\left|z^{j}\right| \leqq q^{2} .
$$


If $p+1 \leqq j \leqq n,(4.5)$ and (b) yield

$$
\left|z_{\gamma}^{j}\right| \leqq 2^{-p} \sum_{v \in C_{\alpha}}\left|\bar{u}_{\gamma}^{j}-\bar{u}_{v}^{j}\right| \leqq q .
$$

Hence we have always

$$
\left\|z_{\gamma}\right\| \leqq q .
$$

Moreover, if $p+1 \leqq j \leqq n,(4.5)$ implies $z^{j}=0$. Thus

$$
\|z\| \leqq q^{2} .
$$

From (4.2) we obtain

$$
\bar{u}_{\gamma}=G\left(\bar{u}_{\gamma}\right)=w_{\alpha}+G^{\prime}\left(x_{\alpha}\right) z_{\gamma}+\left\|z_{\gamma}\right\| \varepsilon\left(z_{\gamma}, x_{\alpha}\right) .
$$

Summing over $\gamma \in C_{\alpha}$ and multiplying by $2^{-p}$ yields

where

$$
w_{\alpha}=2^{-p} \sum_{\gamma \in C_{\alpha}} \bar{u}_{\gamma}-a_{\alpha}-b_{\alpha},
$$

$$
\begin{gathered}
a_{\alpha}=G^{\prime}\left(x_{\alpha}\right) z, \\
b_{\alpha}=2^{-p} \sum_{\gamma \in C_{\alpha}}\left\|z_{\gamma}\right\| \varepsilon\left(z_{\gamma}, x_{\alpha}\right) .
\end{gathered}
$$

From (4.3), (4.6) and (4.7) we obtain the estimates

$$
\left\|a_{\alpha}\right\| \leqq M q^{2}, \quad\left\|b_{\alpha}\right\| \leqq q \varepsilon_{n}(q) .
$$

Suppose now that $\alpha, \alpha^{\prime} \in \Gamma_{p}$ with $\alpha \sim \alpha^{\prime}$. Then $C_{\alpha} \cap C_{\alpha^{\prime}}$ is not empty. Using (b) and the estimates above we obtain

$$
\begin{gathered}
\left\|w_{\alpha}-w_{\alpha^{\prime}}\right\| \leqq 2^{-p}\left(2^{p}-1\right) q+\left\|a_{\alpha}\right\|+\left\|a_{\alpha^{\prime}}\right\|+\left\|b_{\alpha}\right\|+\left\|b_{\alpha^{\prime}}\right\| \\
\leqq\left(1-2^{-p}\right) q+2 M q^{2}+2 q \varepsilon_{n}(q) \leqq\left(1-2^{-p-1}\right) q,
\end{gathered}
$$

as soon as $q \leqq 2^{-p-3} / M$ and $\varepsilon_{n}(q) \leqq 2^{-p-3}$. This proves (3).

4.8. For the next result we introduce some notation. Let $p \geqq 1$ be an integer. For each integer $k$ we let $\mathscr{J}_{k}(p)$ denote the standard subdivision of $R^{p}$ into closed $p$-cubes of side length $2^{k}$. Thus each $Q \in \mathscr{J}_{k}(p)$ contains exactly $2^{p}$ cubes of $\mathscr{J}_{k-1}(p)$. Writing $Q=a_{Q}+2^{k} J^{p}, J^{p}=[0,1]^{p}$, we say that the cube $P_{Q}=a_{Q}+2^{k-1} J^{p} \in \mathscr{J}_{k-1}(p)$ is the principal subcube of $Q$. Let $\mathscr{J}(p)$ be the union of all $\mathscr{J}_{k}(p), k \in Z$. If $Q \in \mathscr{J}_{k}(p)$, we write $k=k(Q)$. If $k(Q)=k(R)$ and $Q \cap R \neq \emptyset$, we write $Q \sim R$. We also define a relation $\cong$ in $\mathscr{J}(p): Q \cong R$ if either $Q \sim R$ or $|k(Q)-k(R)|=1$ and $Q \subset R$ or $R \subset Q$.

4.9. Lemma. For every integer $n \geqq 2$ there is a number $q_{1}=q_{1}(n)>0$ such that for every $q \in\left(0, q_{1}\right]$ there $i s r_{q}>0$ with the following property: Let $1 \leqq p \leqq n-1$ and let $v: \mathscr{J}(p) \rightarrow V_{p}^{0}\left(R^{n}\right)$ be a map such that $\left\|v_{Q}-v_{R}\right\| \leqq r_{q}$ whenever $Q \cong R$. Then there is a map $u: \mathscr{J}(p) \rightarrow V_{n}^{0}\left(R^{n}\right)$ such that

(1) $u_{Q}^{j}=v_{Q}^{j}$ for $1 \leqq j \leqq p$ and $Q \in \mathscr{J}(p)$,

(2) $\left\|u_{Q}-u_{R}\right\| \leqq q$ whenever $Q \cong R$. 
If $Q_{0} \in \mathscr{J}(p)$ and if $u_{0} \in V_{n}^{0}\left(R^{n}\right)$ satisfies the condition $u_{0}^{j}=v_{Q_{0}}^{j}$ for $1 \leqq j \leqq p$, we can choose $u_{Q_{0}}=u_{0}$.

Proof. We may assume $Q_{0}=J^{p}$. Let $q_{0}$ be the number given by Lemma 4.4. We show that the lemma is true for $q_{1}=\min \left(q_{0} / 2,2^{-n-2}\right)$. Suppose $0<q \leqq q_{1}$. Since $V_{n}^{0}\left(R^{n}\right)$ is compact and since the Gram-Schmidt map $G$ is continuous, there is $r_{q} \in\left(0, q^{2}\right]$ such that $r_{q}$ is less than the distance between $V_{n}^{0}\left(R^{n}\right)$ and $\left(R^{n}\right)^{n} \backslash V_{n}\left(R^{n}\right)$ and such that

$$
\|G(x)-y\| \leqq q^{2},
$$

whenever $y \in V_{n}^{0}\left(R^{n}\right), x \in\left(R^{n}\right)^{n}$ and $\|x-y\| \leqq r_{q}$. We claim that the lemma is true with this $r_{q}$.

So let $1 \leqq p \leqq n-1$, and let $v$ be as in the lemma. Suppose that $Q \in \mathscr{J}(p)$ and that $u_{Q} \in V_{n}^{0}\left(R^{n}\right)$ satisfies (1). If $R \in \mathscr{J}(p)$ and $R \cong Q$, we define

$$
x_{R}=\left(v_{R}^{1}, \ldots, v_{R}^{p}, u_{Q}^{p+1}, \ldots, u_{Q}^{n}\right) .
$$

Then $\left\|x_{R}-u_{Q}\right\|=\left\|v_{R}-v_{Q}\right\| \leqq r_{q}$, which implies $x_{R} \in V_{n}\left(R^{n}\right)$. Hence we can form $u_{R}=G\left(x_{R}\right)$. This $u_{R}$ satisfies (1) and

$$
\left\|u_{Q}-u_{R}\right\| \leqq q^{2}
$$

which is stronger than (2). We say that $u_{R}$ is defined directly by $u_{Q}$.

For every integer $k \geqq 1$ we let $\mathscr{J}(p, k)$ denote the family of the cubes $Q \in \mathscr{J}(p)$ which are contained in $2^{k} I^{p}=\left[-2^{k}, 2^{k}\right]^{p}$. Assume that we have defined maps $u(k)$ : $\mathscr{J}(p, k) \rightarrow V_{n}^{0}\left(R^{n}\right)$ satisfying (1) and (2). Since $V_{n}^{0}\left(R^{n}\right)$ is compact, we can apply the diagonal process to find a subsequence $u\left(k_{1}\right), u\left(k_{2}\right), \ldots$ such that for each $Q \in \mathscr{J}(p)$, $u_{Q}\left(k_{j}\right)$ converges to a limit $u_{Q}$ as $j \rightarrow \infty$. Thus we obtain a map $u: \mathscr{J}(p) \rightarrow V_{n}^{0}\left(R^{n}\right)$, which clearly satisfies (1) and (2). Consequently, it suffices to find a map $u: \mathscr{J}(p, k) \rightarrow$ $V_{n}^{0}\left(R^{n}\right)$ satisfying (1) and (2), and for which $u_{Q_{0}}=u_{0}$.

We start with the cube $Q_{0}=J^{p}$ and define $u_{Q_{0}}=u_{0}$. For $Q_{j}=2^{j} J^{p}, 1 \leqq j \leqq k$, we inductively define $u_{Q_{j}}$ directly by $u_{Q_{j-1}}$. Let $\mathscr{K}_{k}$ be the family of the $3^{p}$ cubes $R \in \mathscr{J}(p)$ with $R \sim Q_{k}$. For every $R \in \mathscr{K}_{k}$, we define $u_{R}$ directly by $u_{Q_{k}}$. If $R, R^{\prime} \in \mathscr{K}_{k}$, then

$$
\left\|u_{R}-u_{R^{\prime}}\right\| \leqq\left\|u_{R}-u_{Q_{k}}\right\|+\left\|u_{Q_{k}}-u_{R^{\prime}}\right\| \leqq 2 q^{2} \leqq q .
$$

For every $R \in \mathscr{K}_{k}, R \neq Q_{k}$, we define $u_{P_{R}}$ for the principal subcube $P_{R}$ directly by $u_{R}$. Let $E_{k-1}$ be the convex hull of $\cup\left\{P_{R}: R \in \mathscr{K}_{k}\right\}$, and let $\mathscr{K}_{k-1}$ be the family of the cubes of $\mathscr{J}_{k-1}(p)$ contained in $E_{k-1}$. Suppose that $Q \in \mathscr{K}_{k-1}$ and that $Q$ is not any principal subcube $P_{R}, R \in \mathscr{K}_{k}$. Then $Q$ belongs to a family $\Gamma_{Q}$ of $3^{p}$ cubes in $\mathscr{K}_{k-1}$ such that $\Gamma_{Q}$ is isomorphic in the obvious sense to the Rubik $p$-cube $\Gamma_{p}$ of 4.1 and such that the subfamily $\Gamma_{Q}^{\prime} \subset \Gamma_{Q}$ corresponding to $\Gamma_{p}^{\prime}$ contains only cubes of the form $P_{R}$. Furthermore, if $P_{R}, P_{S} \in \Gamma_{Q}^{\prime}$, (3) implies

$$
\left\|u_{P_{R}}-u_{P_{S}}\right\| \leqq\left\|u_{P_{R}}-u_{R}\right\|+\left\|u_{R}-u_{S}\right\|+\left\|u_{S}-u_{P_{S}}\right\| \leqq 4 q^{2} .
$$


Since $q \leqq 2^{-n-2}<1 / 4$ and since $r_{q} \leqq q^{2}$, we can apply 4.4 to find $u_{Q} \in V_{n}^{0}\left(R^{n}\right)$ satisfying (1) and

$$
\left\|u_{Q}-u_{Q^{\prime}}\right\| \leqq\left(1-2^{-p-1}\right) q \leqq q,
$$

whenever $Q, Q^{\prime} \in \mathscr{K}_{k-1}$ and $Q \sim Q^{\prime}$. The condition (4) of 4.4 guarantees that $u_{Q}$ is independent of the choice of $\Gamma_{Q}$. Furthermore, if $Q \in \mathscr{K}_{k-1}$ and $R \in \mathscr{K}_{k}$ with $Q \cong R$, then $Q \sim P_{R}$, and we obtain

$$
\left\|u_{Q}-u_{R}\right\| \leqq\left\|u_{Q}-u_{P_{R}}\right\|+\left\|u_{P_{R}}-u_{R}\right\| \leqq\left(1-2^{-p-1}\right) q+q^{2} \leqq q,
$$

because $q \leqq 2^{-n-2}<2^{-p-1}$.

We can now proceed in a similar manner to smaller cubes. We describe the next step. For every $R \in \mathscr{K}_{k-1}, R \neq Q_{k-1}$, we define $u_{P_{R}}$ directly by $u_{R}$. Let $E_{k-2}$ be the convex hull of $\cup\left\{P_{R}: R \in \mathscr{K}_{k-1}\right\}$, and let $\mathscr{K}_{k-2}$ be the family of the cubes of $\mathscr{J}_{k-2}(p)$ contained in $E_{k-2}$. If $Q \in \mathscr{K}_{k-2}$ and $Q$ is not of the form $P_{R}, R \in \mathscr{K}_{k-1}$, we can choose the Rubik $p$-cube $\Gamma_{Q}$ as above. If $P_{R}, P_{S} \in \Gamma_{Q}^{\prime}$, then $R \sim S$, and (4.10) and (3) yield

$$
\left\|u_{P_{R}}-u_{P_{S}}\right\| \leqq\left\|u_{P_{R}}-u_{R}\right\|+\left\|u_{R}-u_{S}\right\|+\left\|u_{S}-u_{P_{S}}\right\| \leqq q+2 q^{2} .
$$

Since $q \leqq 2^{-n-2}<2^{-1}, q+2 q^{2} \leqq 2 q \leqq q_{0}$. Since $r_{q} \leqq q^{2} \leqq\left(q+2 q^{2}\right)^{2}$, we can apply 4.4 to find $u_{Q}$ satisfying (1) so that

$$
\left\|u_{Q}-u_{Q^{\prime}}\right\| \leqq\left(q+2 q^{2}\right)\left(1-2^{-p-1}\right),
$$

whenever $Q \sim Q^{\prime}$ in $\mathscr{K}_{k-2}$. Since $q \leqq 2^{-n-2} \leqq 2^{-p-3}$, this implies

$$
\left\|u_{Q}-u_{Q^{\prime}}\right\| \leqq q-q^{2} \leqq q .
$$

If $Q \in \mathscr{K}_{k-2}$ and $R \in \mathscr{K}_{k-1}$ with $Q \cong R$, then $Q \sim P_{R}$, and we obtain

$$
\left\|u_{Q}-u_{R}\right\| \leqq\left\|u_{Q}-u_{P_{R}}\right\|+\left\|u_{P_{R}}-u_{R}\right\| \leqq q-q^{2}+q^{2}=q .
$$

The step is completed.

Continuing in this manner, $u$ will be defined in the family $\cup\left\{\mathscr{K}_{j}: j \leqq k\right\}$, which contains $\mathscr{J}(p, k)$.

4.11. Remarks. 1. Orientation divides $V_{n}^{0}\left(R^{n}\right)$ into two disjoint compact subsets. Replacing $q_{1}$ in Lemma 4.9 by a smaller constant if necessary, we can therefore conclude from (2) that all frames $u_{Q}$ have the same orientation, which can be chosen arbitrarily.

2. In the case $p=n-1$, the results in this section are not really needed in the sequel. On the other hand, Lemmas 4.4 and 4.9 are in this case almost trivial, since if $u \in V_{n}^{0}\left(R^{n}\right), u^{n}$ is uniquely determined up to the sign by $\left(u^{1}, \ldots, u^{n-1}\right)$. 


\section{Extension}

5.1. In this section we prove the main results of the paper, mentioned in the introduction. We shall give the proof of the QS case in detail. In the bilipschitz case, some steps of the proof are obtained as direct corollaries of the QS case, since an $L$-bilipschitz map is $\left(L^{2}-1\right)^{1 / 2}-\mathrm{QS}$. In other steps, we check that the construction gives bilipschitz maps if the given maps are bilipschitz. A direct proof for the bilipschitz case would be somewhat but not much simpler. A notable exception is the reduction of the case $X=S^{p}$ to the case $X=R^{p}$, where we shall give separate proofs in these two categories.

5.2. Triangulation of $R^{n} \backslash R^{p}$. Let $n>p \geqq 1$ be integers. Let $\mathscr{J}(p)$ be the family of $p$-cubes defined in 4.8. Each $Q \in \mathscr{J}(p)$ can be uniquely written as

$$
Q=a_{Q}+\lambda_{Q} J^{p}
$$

where $a_{Q}$ is a vertex of $Q$ and $\lambda_{Q}=2^{k(Q)}$ the side length of $Q$. For each $Q \in \mathscr{J}(p)$ we set

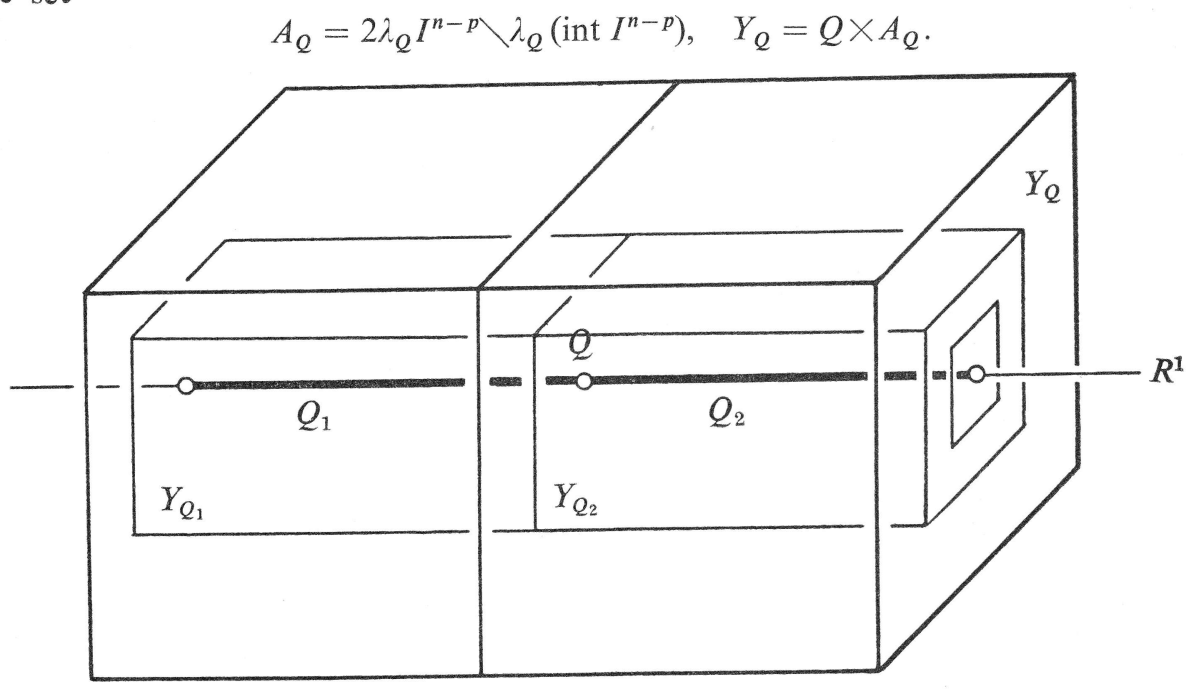

Figure 1.

Then $Y_{Q}$ is a compact polyhedron in $R^{n}$. Figure 1 illustrates the case $n=3, p=1$. Here $Q_{1}, Q_{2}$ are the members of $\mathscr{J}(p)$ with $k\left(Q_{j}\right)=k(Q)-1$ contained in $Q$. To clarify the figure, we have used different scales in $R^{1} \times 0$ and in $0 \times R^{2}$. The collection of the sets $Y_{Q}$ has the following properties:

(1) $\cup\left\{Y_{Q}: Q \in \mathscr{J}(p)\right\}=R^{n} \backslash R^{p}$,

(2) int $Y_{Q}$ int $Y_{R}=\emptyset$ for $Q \neq R$,

(3) $Y_{Q} \cap Y_{R} \neq \emptyset$ if and only if $|k(Q)-k(R)| \leqq 1$ and $Q \cap R \neq \emptyset$. 
It is fairly obvious that there is a triangulation $W$ of $R^{n} \backslash R^{k}$ such that each $Y_{Q}$ is the underlying space of a subcomplex $W_{Q}$ of $W$ and such that for $Q, R \in \mathscr{J}(p)$, the natural similarity map $Y_{Q} \rightarrow Y_{R}$ maps $W_{Q}$ onto $W_{R}$. In fact, all that is essential in the proofs is that (1) the flatness $d(\Delta) / b(\Delta)$ of the $n$-simplexes $\Delta$ of $W$ is bounded, and (2) the diameter of an $n$-simplex of $W$ meeting $Y_{Q}$ has the same order of magnitude as $\lambda_{Q}$. We give a construction of $W$, which is not the most economical one.

The boundary of $A_{Q}$ consists of the sets $B_{Q}=\lambda_{Q} \partial I^{n-p}$ and $2 B_{Q}$. These sets have natural structures as cell complexes. The natural product structure of $A_{Q} \approx B_{Q} \times I$ defines a cell complex structure for $A_{Q}$. Taking the cartesian product we obtain a cell complex $C_{Q}$ with $\left|C_{Q}\right|=Q \times A_{Q}=Y_{Q}$. We replace the subcomplex of $C_{Q}$ corresponding to $Q \times B_{Q}$ by the subdivision induced by the subdivision of $Q$ into $2^{p}$ cubes of side length $\lambda_{Q} / 2$. We get a subdivision $C_{Q}^{\prime}$ of $C_{Q}$, which is not a cell complex. The union $C=\cup\left\{C_{Q}^{\prime}: Q \in \mathscr{J}(p)\right\}$ is a collection of cells with the properties:

(1) $\cup C=R^{n} \backslash R^{k}$.

(2) The members of $C$ have disjoint cell interiors.

(3) The boundary of each cell is a union of some members of $C$.

The 1-skeleton $C^{1}$ of $C$ is a simplicial complex. For every 2-cell $E \in C$ we choose an interior point $b_{E}$ such that the natural similarity mappings $Y_{Q} \rightarrow Y_{R}$ map the points $b_{E}$ onto each other. Since $\partial E$ is triangulated, we can triangulate $E$ by the cone construction from $b_{E}$. This gives a simplicial subdivision of the 2-skeleton $C^{2}$. Proceeding similarly to cells of higher dimensions we obtain the triangulation $W$.

5.3. Theorem. For every integer $n \geqq 2$ there is a number $L_{0}=L_{0}(n)>1$ with the following property: Let $1 \leqq p \leqq n-1$, let $1 \leqq L \leqq L_{0}$, and let $f: R^{p} \rightarrow R^{n}$ be $L$ bilipschitz. Then $f$ has an extension to an $L_{1}$-bilipschitz homeomorphism $g: R^{n} \rightarrow R^{n}$ where $L_{1}=L_{1}(L, n) \rightarrow 1$ as $L \rightarrow 1$.

5.4. Theorem. For every integer $n \geqq 2$ there is a number $s_{0}=s_{0}(n)>0$ with the following property: Let $1 \leqq p \leqq n-1$, let $0 \leqq s \leqq s_{0}$, and let $f: R^{p} \rightarrow R^{n}$ be $s-\mathrm{QS}$. Then f has an extension to an $s_{1}$-QS homeomorphism $g: R^{n} \rightarrow R^{n}$ where $s_{1}=s_{1}(s, n) \rightarrow 0$ as $s \rightarrow 0$.

Proof. As mentioned in 5.1, we shall prove Theorem 5.4 in detail and obtain Theorem 5.3 by taking care that the construction gives bilipschitz maps if $f$ is bilipschitz.

Let $p \in[1, n-1]$ be an integer. It suffices to find $s_{0}>0$ such that 5.4 is true with this $p$. Let $s_{2}=s_{2}(p)$ be the number given by 3.11. Let $0 \leqq s \leqq s_{2}$, and let $f: R^{p} \rightarrow R^{n}$ be $s$-QS. In the bilipschitz case, we set $L_{2}=\left(1+s_{2}^{2}\right)^{1 / 2}$ and assume that $1 \leqq L \leqq L_{2}$ and that $f$ is $L$-bilipschitz. Then $f$ is $s$-QS with $s=\left(L^{2}-1\right)^{1 / 2} \leqq s_{2}$.

Using the notation of 4.8 and 5.2 , we consider a cube $Q=a_{Q}+\lambda_{Q} J^{p} \in \mathscr{J}(p)$. For every $j \in[1, p]$ we set

$$
w_{Q}^{j}=f\left(a_{Q}+\lambda_{Q} e_{j}\right)-f\left(a_{Q}\right) .
$$


It follows from 3.11 that $w_{Q} \in V_{p}\left(R^{n}\right)$. Hence we can apply the Gram-Schmidt process and obtain

$$
v_{Q}=G\left(w_{Q}\right) \in V_{p}^{0}\left(R^{n}\right) .
$$

We define the orthogonal map $\varphi_{Q}: R^{p} \rightarrow R^{n}$ by

and the numbers

$$
\varphi_{Q} e_{j}=v_{Q}^{j}
$$

$$
\varrho_{Q}=\left|w_{Q}^{1}\right|, \quad \mu_{Q}=\varrho_{Q} / \lambda_{Q} .
$$

Let $h_{Q}: R^{p} \rightarrow R^{n}$ be the similarity defined by

$$
h_{Q}(x)=f\left(a_{Q}\right)+\mu_{Q} \varphi_{Q}\left(x-a_{Q}\right) .
$$

If $s=0, h_{Q}=f$. If $s>0, h_{Q}$ is an approximation of $f$ near $a_{Q}$. More precisely, if $y \in R^{p}$ and $\left|y-a_{Q}\right| \leqq r \lambda_{Q}$, then

$$
\left|f(y)-h_{Q}(y)\right| \leqq \varepsilon(s, p, r) \varrho_{Q},
$$

where $\varepsilon$ is the function given by Lemma 3.10. To prove this, we may assume that $a_{Q}=0=f\left(a_{Q}\right)$. Define $f_{1}: R^{p} \rightarrow R^{n}$ by $f_{1}(x)=f\left(\lambda_{Q} x\right)$. Then $f_{1}$ is $s-\mathrm{QS}$ and $\left|f_{1}\left(e_{1}\right)\right|=$ $\varrho_{Q}$. Choose a rotation $\psi$ of $R^{n}$ such that $\psi f_{1}\left(e_{j}\right) \in R_{+}^{j}$ for $1 \leqq j \leqq p$. Since $\psi f_{1}$ is $s-\mathrm{QS}$ and since $\psi v_{Q}^{j}=e_{j}$, (5.5) follows easily from 3.10.

If suffices to find a number $q_{0}=q_{0}(n)>0$ such that for every $q \in\left(0, q_{0}\right]$ there is $s=s(q, n)>0$ such that every $s-\mathrm{QS}$ map $f: R^{p} \rightarrow R^{n}$ has an $s_{1}-\mathrm{QS}$ extension $g: R^{n} \rightarrow R^{n}$ where $s_{1}=s_{1}(q, n) \rightarrow 0$ as $q \rightarrow 0$. In the bilipschitz case, we shall find $L=L(q, n)>1$ such that every $L$-bilipschitz map $f: R^{p} \rightarrow R^{n}$ has an $L_{1}$-bilipschitz extension $g: R^{n} \rightarrow R^{n}$ where $L_{1}=L_{1}(q, n) \rightarrow 1$ as $q \rightarrow 0$.

Let $0<q<q_{1}$ where $q_{1}$ is given by 4.9. Additional restrictions for $q$ will be given later. Let $r_{q}>0$ be the number given by 4.9. From (5.5) it easily follows that there is $s_{3}=s_{3}(q, n) \leqq s_{2}$ such that $s \leqq s_{3}$ implies

$$
\left\|v_{Q}-v_{R}\right\| \leqq r_{q}
$$

whenever $Q \cong R$ in $\mathscr{J}(p)$. From now on, we assume $s \leqq s_{3}$.

Let $u: \mathscr{J}(p) \rightarrow V_{n}^{0}\left(R^{n}\right)$ be the map given by Lemma 4.9. We may assume that the frames $u_{Q}$ are positively oriented, that is, they have the same orientation as the standard basis $\left(e_{1}, \ldots, e_{n}\right)$. We extend the maps $\varphi_{Q}$ and $h_{Q}$ to a rotation $\varphi_{Q}: R^{n} \rightarrow R^{n}$ and to a sense-preserving similarity $h_{Q}: R^{n} \rightarrow R^{n}$ by

$$
\varphi_{Q} e_{j}=u_{Q}^{j}, \quad h_{Q}(x)=f\left(a_{Q}\right)+\mu_{Q} \varphi_{Q}\left(x-a_{Q}\right) .
$$

For each vertex $b$ of the triangulation $W$ of $R^{n} \backslash R^{p}$, we choose $Q(b) \in \mathscr{J}(p)$ such that $b \in Y_{Q(b)}$. Set $h_{b}=h_{Q(b)}$ and $g(b)=h_{b}(b)$. Extend $g$ to $R^{n} \backslash R^{p}$ so that $g$ is affine on every simplex of $W$, and define $g \mid R^{p}=f$. We claim that this map $g: R^{n} \rightarrow R^{n}$ is the desired extension of $f$ provided that $q$ and $s$ are sufficiently small. 
We first show that if $Q, R \in \mathscr{J}(p)$ with $Q \cap R \neq \emptyset$ and $|k(Q)-k(R)| \leqq 1$, then (5.6)

$$
\left|\mu_{Q}-\mu_{R}\right|<4 \varepsilon \mu_{Q}
$$

where $\varepsilon=\varepsilon(s, p, 3 n)$.

Since (5.5) is true for all $y \in Q \cup R$ with $r=3 n$, we have

$$
\varrho_{R}=\left|h_{Q}\left(a_{R}+\lambda_{R} e_{1}\right)-h_{Q}\left(a_{R}\right)\right|+x=\mu_{Q} \lambda_{R}+x
$$

where $|x| \leqq 2 \varepsilon \varrho_{Q}$. Since $\lambda_{Q} \leqq 2 \lambda_{R}$, this implies (5.6).

We next estimate $\left|g-h_{Q}\right|$ in $Y_{Q}$. Let $b \in Y_{Q}$ be a vertex of $W$, and set $R=Q(b)$. Since $b \in Y_{Q} \cap Y_{R},|k(Q)-k(R)| \leqq 1$ and $Q \cap R \neq \emptyset$. Hence either $Q \cong R$ or $Q \cong Q^{\prime} \cong R$ for some $Q^{\prime} \in \mathscr{J}(p)$. Consequently, $\left\|u_{Q}-u_{R}\right\| \leqq 2 q$. This implies $\left|\varphi_{Q}-\varphi_{R}\right| \leqq 2 n q$; for linear maps we use the sup-norm. Clearly $\left|b-a_{R}\right| \leqq 2 n \lambda_{R} \leqq 4 n \lambda_{Q}$ and $\left|a_{R}-a_{Q}\right| \leqq$ $d(Q)+d(R) \leqq 3 n \lambda_{Q}$. Hence (5.5) gives

$$
\begin{aligned}
\left|g(b)-h_{Q}(b)\right| & \leqq\left|f\left(a_{R}\right)-h_{Q}\left(a_{R}\right)\right|+\left|\mu_{R} \varphi_{R}-\mu_{Q} \varphi_{Q}\right|\left|b-a_{R}\right| \\
& \leqq \varepsilon \varrho_{Q}+4 n \lambda_{Q}\left|\mu_{R} \varphi_{R}-\mu_{Q} \varphi_{Q}\right| .
\end{aligned}
$$

Here (5.6) implies

$$
\left|\mu_{R} \varphi_{R}-\mu_{Q} \varphi_{Q}\right| \leqq \mu_{Q}\left|\varphi_{Q}-\varphi_{R}\right|+\left|\mu_{Q}-\mu_{R}\right|\left|\varphi_{R}\right| \leqq 2 n q \mu_{Q}+4 \varepsilon \mu_{Q} .
$$

Combining these estimates yields

$$
\left|g(b)-h_{Q}(b)\right| \leqq\left(\varepsilon+8 n^{2} q+16 n \varepsilon\right) \varrho_{Q} .
$$

Choose $s=s(q, n)>0$ such that

$$
\varepsilon(s, p, 3 n) \leqq n q / 17 \text {. }
$$

In the bilipschitz case, we choose $L=L(q, n)=\left(1+s^{2}\right)^{1 / 2}$. Since $g-h_{Q}$ is affine on every simplex of $W$, we obtain

$$
\left\|g-h_{Q}\right\|_{Y_{Q}} \leqq 9 n^{2} q \varrho_{Q}
$$

for every $Q \in \mathscr{J}(p)$.

As a PL map, $g$ is continuous in $R^{n} \backslash R^{p}$. We next show that $g$ is continuous also at an arbitrary point $z \in R^{p}$. Let $\varepsilon_{1}>0$. Since $f$ is continuous, there is $\delta>0$ such that $|f(y)-f(z)|<\varepsilon_{1} /(4 n+1)$ whenever $|y-z|<\delta$. Choose $\delta_{1} \in(0, \delta)$ such that if $\Delta$ is an $n$-simplex of $W$ meeting $B^{n}\left(z, \delta_{1}\right)$ and if $b \in \Delta^{0}$, then $Q(b) \subset B^{p}(z, \delta)$. We show that $|g(x)-g(z)|<\varepsilon_{1}$ for every $x \in B^{n}\left(z, \delta_{1}\right) \backslash R^{p}$. Choose an $n$-simplex $\Delta \in W$ containing $x$. It suffices to show that $|g(b)-g(z)|<\varepsilon_{1}$ for each vertex $b$ of $\Delta$. The cube $Q=Q(b)$ is contained in $B^{n}(z, \delta)$. Since $g(b)=h_{Q}(b)$, we obtain

$$
\begin{aligned}
& |g(b)-g(z)| \leqq\left|h_{Q}(b)-h_{Q}\left(a_{Q}\right)\right|+\left|f\left(a_{Q}\right)-f(z)\right| \\
& \leqq \mu_{Q}\left|b-a_{Q}\right|+\varepsilon_{1} /(4 n+1) \leqq 2 n \varrho_{Q}+\varepsilon_{1} /(4 n+1) .
\end{aligned}
$$

Since $\varrho_{Q} \leqq d(f Q)<2 \varepsilon_{1} /(4 n+1)$, the right-hand side is less than $\varepsilon_{1}$. Hence $g$ is continuous at $z$. 
Let $\Delta \in W$ be an $n$-simplex. Choose $Q \in \mathscr{J}(p)$ with $\Delta \subset Y_{Q}$. To estimate the dilatation and the bilipschitz constant of $g \mid \Delta$, we want to apply 3.5 with the substitution $f \mapsto g \mid \Delta, h \mapsto h_{Q}$. From the construction of $W$ it follows that there is $M=M(n) \geqq 1$ such that $b(\Delta) \geqq \lambda_{Q} / M$. By (5.7), this implies that we may apply 3.5 with $\alpha=$ $9 n^{2}(n+1) M q$ provided that $\alpha \leqq 1 / 2$. This gives the new restriction

$$
q \leqq \frac{1}{18 n^{2}(n+1) M}
$$

in addition to $q \leqq q_{1}$. By $3.5, g \mid \Delta$ is sense-preserving, and

$$
H(g \mid \Delta) \leqq\left(1+18 n^{2}(n+1) M q\right)^{2}=H_{1}(q, n)
$$

where $H_{1}(q, n) \rightarrow 1$ as $q \rightarrow 0$.

In the bilipschitz case, observe first that $1 / L \leqq \mu_{Q}=L_{h} \leqq L$. Hence 3.5 implies that $g \mid \Delta$ is $L_{1}$-bilipschitz with

$$
L_{1}=L_{1}(q, n)=L\left(1+18 n^{2}(n+1) M q\right) .
$$

To complete the proof of Theorems 5.3 and 5.4, it suffices to show that $g: R^{n} \rightarrow R^{n}$ is injective. Indeed, $f$ is then $H_{1}^{n-1}$-quasiconformal by [V $\ddot{a}_{1}, 34.2$ and 35.1], and Theorem 5.4 follows from 2.6. In particular, $g R^{n}=R^{n}$. In the bilipschitz case, it follows then almost immediately that $g$ is $L_{1}$-bilipschitz.

Let $Q \in \mathscr{J}(p)$, and set

$$
E_{Q}=\cup\{R: R \sim Q\}, \quad Z_{Q}=E_{Q} \times 2 \lambda_{Q} I^{n-p} .
$$

Thus $Z_{Q}$ is the convex hull of $\cup\left\{Y_{R}: R \sim Q\right\}$. We shall first prove that

$$
\left\|g-h_{Q}\right\|_{Z_{Q}} \leqq 24 n^{2} q \varrho_{Q} .
$$

Let $R \cong Q$ with $k(R) \leqq k(Q)$. Then $\left|\varphi_{R}-\varphi_{Q}\right| \leqq n q$ and $\left|x-a_{R}\right| \leqq 3 n \lambda_{Q}$ for all $x \in Z_{Q}$. Since

$$
h_{Q}(x)-h_{R}(x)=h_{Q}\left(a_{R}\right)-f\left(a_{R}\right)+\left(\mu_{Q} \varphi_{Q}-\mu_{R} \varphi_{R}\right)\left(x-a_{R}\right),
$$

we obtain as in the proof of (5.7),

$$
\left\|h_{Q}-h_{R}\right\|_{z_{Q}} \leqq 4 n^{2} q \varrho_{Q} .
$$

Let $x \in Z_{Q} \backslash R^{p}$. Then there is a finite sequence $R_{1} \supset \ldots \supset R_{t}$ in $\mathscr{J}(p)$ such that $Q \sim R_{1}, k\left(R_{j+1}\right)=k\left(R_{j}\right)-1$, and $x \in Y_{R_{t}}$. By (5.6), $\varrho_{R_{1}} \leqq 2 \beta \varrho_{Q}$ and $\varrho_{R_{j+1}} \leqq \beta \varrho_{R_{j}}$ where $\beta=(1+4 \varepsilon) / 2$. Since $n \geqq 2$ and $M \geqq 1$, (5.8) gives the rough estimate $\varepsilon \leqq$ $n q / 17<1 / 36$, which implies $\beta \leqq 5 / 9$. Applying (5.10) with the substitutions $(Q, R) \mapsto\left(Q, R_{1}\right)$ and $(Q, R) \mapsto\left(R_{j}, R_{j+1}\right)$ we obtain

By (5.7),

$$
\begin{gathered}
\left|h_{Q}(x)-h_{R_{t}}(x)\right| \leqq 4 n^{2} q \varrho_{Q}\left(1+2 \beta+2 \beta^{2}+\ldots+2 \beta^{t-1}\right) \\
\leqq 4 n^{2} q \varrho_{Q}(1+2 \beta /(1-\beta)) \leqq 14 n^{2} q \varrho_{Q} .
\end{gathered}
$$

$$
\left|g(x)-h_{R_{t}}(x)\right| \leqq 9 n^{2} q \varrho_{R_{t}} \leqq 10 n^{2} q \varrho_{Q},
$$


whence

$$
\left|g(x)-h_{Q}(x)\right| \leqq 24 n^{2} q \varrho_{Q}
$$

for $x \in Z_{Q} \backslash R^{p}$. By continuity, this inequality also holds for $x \in Z_{Q} \cap R^{p}$, and we obtain (5.9).

For $Q \in \mathscr{J}(p)$ let $Y_{Q}^{\prime}$ be the union of all $Y_{R}$ such that $Y_{R} \cap Y_{Q} \neq \emptyset$ and $k(R) \leqq$ $k(Q)$. Then

$$
d\left(Y_{Q}, Z_{Q} \backslash Y_{Q}^{\prime}\right)=\lambda_{Q} / 2 .
$$

Suppose that $x$ and $y$ are distinct points in $R^{n}$. We want to show that $g(x) \neq g(y)$. Since $g \mid R^{p}=f$ is injective, we may assume that $x \notin R^{p}$. Choose $Q \in \mathscr{J}(p)$ such that $x \in Y_{Q}$. We may assume that either $y \in R^{p}$ or $y \in Y_{S}$ for some $S$ with $k(S) \leqq k(Q)$.

Case 1. $y \notin Y_{Q}^{\prime}$. Now there is a unique sequence $Q=R_{0} \subset R_{1} \subset \ldots$ of cubes of $\mathscr{J}(p)$ such that $k\left(R_{j+1}\right)=k\left(R_{j}\right)+1$. Let $t$ be the smallest number such that $y \in Z_{R_{t}}$, and set $R=R_{t}$. Then $|x-y| \geqq \lambda_{R} / 2$. Thus

$$
\left|h_{R}(x)-h_{R}(y)\right|=\mu_{R}|x-y| \geqq \varrho_{R} / 2 .
$$

Hence (5.9) implies that $g(x) \neq g(y)$ if

$$
q \leqq \frac{1}{100 n^{2}} .
$$

Case 2. $y \in Y_{Q}^{\prime}$. Let $W_{Q}^{\prime}$ be the subcomplex of $W$ for which $\left|W_{Q}^{\prime}\right|=Y_{Q}^{\prime}$. Let $r_{0}\left(W_{Q}^{\prime}\right)$ be the number given by Lemma 3.6. We can choose $r_{0}\left(W_{Q}^{\prime}\right)=\gamma_{0} \lambda_{Q}$ for some $\gamma_{0}=\gamma_{0}(n)>0$. Since $x, y \in Y_{Q}^{\prime} \subset Z_{Q}$, it follows from (5.9) and from 3.6 that $g(x) \neq$ $g(y)$ if

$$
q \leqq \frac{\gamma_{0}}{24 n^{2}} .
$$

The proof of Theorems 5.3 and 5.4 is completed.

5.11. Remark. Suppose that $f$ in 5.3 or in 5.4 satisfies the conditions $f(0)=0$, $f\left(e_{1}\right)=e_{1}$, and $f\left(e_{j}\right) \in R_{+}^{j}$ for $2 \leqq j \leqq p$. Let $Q_{0}=J^{p}$. The $p$-frame $v_{Q_{0}}$ in the proof is then the standard frame $\left(e_{1}, \ldots, e_{p}\right)$. By 4.9 , we can choose $u_{Q_{0}}=\left(e_{1}, \ldots, e_{n}\right)$. Furthermore, we can choose $Q(b)=Q_{0}$ whenever $b \in Y_{Q_{0}}$. This implies that $g \mid Y_{Q_{0}}=\mathrm{id}$. In particular, $g\left(e_{j}\right)=e_{j}$ for $p+1 \leqq j \leqq n$.

5.12. Explicit bounds. Since the proof of 5.3 contains several indirect arguments, it does not give any explicit bounds for $L_{0}(n)$ or for $L_{1}(L, n)$. It is possible, however, to replace these arguments by elementary geometric and trigonometric considerations, especially in low dimensions. For example, one can choose $\varepsilon(s, 1,1)=10 s^{1 / 2}$, $0 \leqq s \leqq 1$, for the function $\varepsilon(s, p, r)$ of 3.10 . We have shown that in the case $p=1$, $n=2$, a slight modification of the proof of 5.3 gives the bound $L_{0}=1+10^{-5}$, and for $L=1+\varepsilon \leqq L_{0}$ the bound

$$
L_{1}=1+100 \varepsilon^{1 / 2}
$$


However, in this case no bound is actually needed for $L_{0}$, since every bilipschitz $f: R^{1} \rightarrow R^{2}$ can be extended to a bilipschitz $g: R^{2} \rightarrow R^{2} \quad\left[\mathrm{Tu}_{2}, \mathrm{JK}, \mathrm{Ge}_{2}, \mathrm{La}\right.$. On the other hand, the bound $1+100 \varepsilon^{1 / 2}$ has the correct order, since the best possible bound for small $\varepsilon$ must be asymptotically at least $1+2 \varepsilon^{1 / 2} / \pi$. To see this, let $0<\alpha<\pi / 2$ and define $f: R^{1} \rightarrow R^{2}$ by

Then

$$
f(x)=\left\{\begin{array}{lll}
x & \text { for } & x \leqq 0 \\
x e^{i \alpha} & \text { for } & x \geqq 0
\end{array}\right.
$$

$$
|x-y| / L \leqq|f(x)-f(y)| \leqq|x-y|
$$

for all $x, y \in R^{1}$, where

$$
L=(1-(1-\cos \alpha) / 2)^{-1 / 2} .
$$

Hence the map $f_{1}: R^{1} \rightarrow R^{2}$, defined by $f_{1}(x)=L^{1 / 2} f(x)$, is $L^{1 / 2}$-bilipschitz.

Suppose that $g: R^{2} \rightarrow R^{2}$ is an $L_{1}$-bilipschitz extension of $f_{1}$. Suppose, for example, that $g$ is sense-preserving. Let $\gamma$ be the circular arc $\left\{e^{i \varphi}: \alpha \leqq \varphi \leqq \pi\right\}$. Then $g^{-1} \gamma$ is an arc in the upper half-plane with end points $L^{-1 / 2}$ and $-L^{-1 / 2}$. Moreover, $g^{-1} \gamma$ does not intersect the disc $B^{2}\left(1 / L_{1}\right)$. Hence the length $l\left(g^{-1} \gamma\right)$ of $g^{-1} \gamma$ satisfies the inequality

$$
l\left(g^{-1} \gamma\right) \geqq \pi / L_{1} .
$$

Since $l\left(g^{-1} \gamma\right) \leqq L_{1} l(\gamma)=L_{1}(\pi-\alpha)$, we obtain

$$
L_{1} \geqq(1-\alpha / \pi)^{-1 / 2} .
$$

Write $L^{1 / 2}=1+\varepsilon$ and assume that $\varepsilon$ is small. Using the first order terms of the Taylor expansions, we get $\alpha \approx 4 \varepsilon^{1 / 2}$, and the lower bound for $L_{1}$ is $\approx 1+2 \varepsilon^{1 / 2} / \pi$.

5.13. Extension from a sphere. We shall next show that Theorems 5.3 and 5.4 remain true if $R^{p}$ is replaced by the sphere $S^{p}$. By the stereographic projection, we shall reduce the problem to the previous case. To simplify notation, we replace $S^{p}$ by the sphere

$$
S=S^{p}\left(e_{p+1} / 2,1 / 2\right)
$$

Let $\bar{R}^{n}=R^{n} \cup\{\infty\}$ be the one-point compactification of $R^{n}$, and let $u: \bar{R}^{n} \rightarrow \bar{R}^{n}$ be the inversion $u(x)=x /|x|^{2}$. Then $u$ maps $S \backslash\{0\}$ onto the $p$-plane

The formula

$$
T=R^{p}+e_{p+1} \text {. }
$$

$$
|u(x)-u(y)|=\frac{|x-y|}{|x||y|}
$$

is true for all $x, y \in R^{n} \backslash\{0\}$.

Let $f: S \rightarrow R^{n}$ be either $L$-bilipschitz or $s-$ QS for some $L$ or $s$. Performing an auxiliary isometry of $R^{n}$, we may assume $f(0)=0$. In the QS case, we may also assume 
$f\left(e_{p+1}\right)=e_{p+1}$. Let $E=\left\{0, e_{1}, \ldots, e_{p}\right\}+e_{p+1} \subset T$. Replacing $f$ by $\psi f$ with a suitable isometry $\psi$ of $R^{n}$, we may assume that

$$
\|f-\mathrm{id}\|_{u E} \leqq \varepsilon_{1}(L, n) \quad \text { or } \quad\|f-\mathrm{id}\|_{u E} \leqq \varepsilon_{1}^{\prime}(s, n)
$$

in the bilipschitz and the QS case, respectively, where $\varepsilon_{1}(L, n) \rightarrow 0$ as $L \rightarrow 1$ and $\varepsilon_{1}^{\prime}(s, n) \rightarrow 0$ as $s \rightarrow 0$. This can be proved by an easy compactness argument, cf. the proof of 3.9. In fact, one could replace $u E$ by $S$ in (5.15), but this stronger fact will not be needed.

Since $f(0)=0, f^{\prime}=u f u$ defines a map $f^{\prime}: T \rightarrow R^{n}$. We want to apply 5.3 or 5.4 to $f^{\prime}$. For this we must show that $f^{\prime}$ satisfies the hypothesis of the appropriate theorem. In the bilipschitz case this is easy:

5.16. Lemma. If $0 \in X \subset R^{n}$ and if $f: X \rightarrow R^{n}$ is L-bilipschitz with $f(0)=0$, then the map $f^{\prime}=u f u: u[X \backslash\{0\}] \rightarrow R^{n}$ is $L^{3}$-bilipschitz.

Proof. This follows by direct computation from (5.14), cf. [ $\left.\mathrm{Ge}_{2}, \mathrm{Lemma} 8\right]$.

5.17. Theorem. Theorem 5.3 remains true if $R^{p}$ is replaced by $S^{p}$.

Proof. Let $L_{0}=L_{0}(n)$ and $L_{1}(L, n)$ be the numbers given by 5.3. Assume that $f: S \rightarrow R^{n}$ is $L$-bilipschitz and that $f$ is normalized as in 5.13. From (5.15) it follows that

$$
\left\|f^{\prime}-\mathrm{id}\right\|_{E} \leqq \varepsilon_{2}(L, n),
$$

where $\varepsilon_{2}(L, n) \rightarrow 0$ as $L \rightarrow 1$. Consequently, there is $L_{0}^{\prime}=L_{0}^{\prime}(n)>1$ such that if $L \leqq L_{0}^{\prime}$, we can choose a linear map $\alpha: R^{n} \rightarrow R^{n}$ such that $\alpha f^{\prime}\left(e_{p+1}\right)=e_{p+1}$, $\alpha f^{\prime}\left(e_{p+1}+e_{j}\right)=e_{p+1}+e_{j}$ for $1 \leqq j \leqq p$, and such that $\alpha$ is $L_{2}$-bilipschitz where $L_{2}=$ $L_{2}(L, n) \rightarrow 1$ as $L \rightarrow 1$. Choose $L_{0}^{\prime \prime}=L_{0}^{\prime \prime}(n)>1$ such that $L_{0}^{\prime \prime} \leqq L_{0}^{1 / 4}, L_{0}^{\prime \prime} \leqq L_{0}^{\prime}$, and $L_{2}(L, n) \leqq L_{0}^{1 / 4}$ for $L \leqq L_{0}^{\prime \prime}$. If $L \leqq L_{0}^{\prime \prime}, 5.16$ implies that $\alpha f^{\prime}$ is $L_{2} L^{3}$-bilipschitz. Since $L_{2} L^{3} \leqq L_{0}$, it follows from 5.3 that we can extend $\alpha f^{\prime}: T \rightarrow R^{n}$ to an $L_{1}$-bilipschitz homeomorphism $g^{\prime \prime}: R^{n} \rightarrow R^{n}, L_{1}=L_{1}\left(L_{2} L^{3}, n\right)$. By 5.11, we can choose $g^{\prime \prime}(0)=0$. Then $g^{\prime}=a^{-1} g^{\prime \prime}: R^{n} \rightarrow R^{n}$ is an $L_{1} L_{2}$-bilipschitz extension of $f^{\prime}$, and $g^{\prime}(0)=0$. Setting $g=u g^{\prime} u$ we obtain an $L_{1}^{\prime}$-bilipschitz extension $g: R^{n} \rightarrow R^{n}$ of $f$, where $L_{1}^{\prime}=\left(L_{1} L_{2}\right)^{3}=L_{1}^{\prime}(L, n) \rightarrow 1$ as $L \rightarrow 1$.

5.18. We now turn to the QS case. Unfortunately, we have not found a simple proof of the QS version of Lemma 5.16. In what follows, we assume that $f: S \rightarrow R^{n}$ is $s-\mathrm{QS}$ and that $f \mid\left\{0, e_{p+1}\right\}=\mathrm{id}$. Then $f$ induces the map $f^{\prime}: T \rightarrow R^{n}$ as in 5.13. We make the following notational convention: Whenever we consider a triple of distinct points $a, b, x$ in $T$, we set

$$
t=\frac{|a-x|}{|b-x|}, \quad t^{\prime}=\frac{\left|f^{\prime}(a)-f^{\prime}(x)\right|}{\left|f^{\prime}(b)-f^{\prime}(x)\right|} .
$$


5.19. Lemma. Let $a, b, x$ be distinct points in $T$ such that $t|b| /|a| \leqq 1 / s$ and $|a| /|b| \leqq 1 / s$. Then

$$
t^{\prime} \leqq t+s\left(t \frac{|b|}{|a|}+\frac{|a|}{|b|}+s\right) .
$$

Proof. Since $f(0)=0$ and $|u(b)| /|u(a)|=|a| /|b| \leqq 1 / s$, we have

Next, since

$$
\frac{|f(u(b))|}{|f(u(a))|} \leqq \frac{|a|}{|b|}+s .
$$

$$
\begin{gathered}
\frac{|u(a)-u(x)|}{|u(b)-u(x)|}=t \frac{|b|}{|a|} \leqq \frac{1}{s}, \\
\frac{|f(u(a))-f(u(x))|}{|f(u(b))-f(u(x))|} \leqq t \frac{|b|}{|a|}+s .
\end{gathered}
$$

The lemma follows from (5.14).

5.20. Lemma. Let $M \geqq 1$ and let $a, b, x$ be distinct points in $T$ such that $t \leqq s^{-1 / 2}, s \leqq M^{-2}$ and $|a||M \leqq| b|\leqq M| a \mid$. Then

$$
t^{\prime} \leqq t+(2 M+1) s^{1 / 2} .
$$
implies

Proof. We have $|a| /|b| \leqq M \leqq M^{2} \leqq 1 / s$ and $t|b| /|a| \leqq s^{-1 / 2} M \leqq 1 / s$. Thus 5.19

$$
t^{\prime} \leqq t+s(M t+M+s) \leqq t+s^{1 / 2}(2 M+1) .
$$

5.21. Lemma. Suppose that $y, z \in T$ with $|y| /|z| \leqq 1 / s$. Then

$$
\frac{\left|f^{\prime}(y)\right|}{\left|f^{\prime}(z)\right|} \leqq \frac{|y|}{|z|}+s \text {. }
$$

Proof. Since $f(0)=0$ and $|u(z)| /|u(y)| \leqq 1 / s$, the lemma follows from the $s$ quasisymmetry of $f$.

5.22. Lemma. There is $s_{0}^{*}=s_{0}^{*}(n)>0$ such that if $0 \leqq s \leqq s_{0}^{*}$, then $f^{\prime}$ is $s^{\prime}-\mathrm{QS}$ where $s^{\prime}=s^{\prime}(s, n) \rightarrow 0$ as $s \rightarrow 0$.

Proof. Suppose that the lemma is false. By 2.4, there is a number $\delta \in(0,1)$, a sequence of $s_{k}$-QS maps $f_{k}: S \rightarrow R^{n}$, and a sequence of triples of distinct points $a_{k}, b_{k}, x_{k} \in T$ such that (1) $s_{k} \rightarrow 0$, (2) $f_{k} \mid\left\{0, e_{p+1}\right\}=\mathrm{id}$, (3) with the obvious notation, we have

$$
\delta \leqq t_{k} \leqq 1 / \delta, \quad t_{k}^{\prime} \geqq t_{k}+\delta .
$$

Passing to a subsequence we may assume that $\alpha_{k}=\left|a_{k}\right| /\left|x_{k}\right| \rightarrow \alpha, \beta_{k}=\left|b_{k}\right| /\left|x_{k}\right| \rightarrow \beta$, $\gamma_{k}=\left|a_{k}\right| /\left|b_{k}\right| \rightarrow \gamma$ with $\alpha, \beta, \gamma \in[0, \infty]$.

If $0<\gamma<\infty$, there is $M \geqq 1$ such that $\left|a_{k}\right| / M \leqq\left|b_{k}\right| \leqq M\left|a_{k}\right|$ for all $k$. Since $t_{k} \leqq 1 / \delta$, we have $t_{k} \leqq s_{k}^{-1 / 2}$ for sufficiently large $k$. Since $s_{k} \leqq M^{-2}$ for large $k$, 
5.20 implies

$$
t_{k}+\delta \leqq t_{k}^{\prime} \leqq t_{k}+s_{k}^{1 / 2}(2 M+1),
$$

which gives a contradiction as $k \rightarrow \infty$.

We may thus assume that $\gamma=0$ or $\gamma=\infty$. Since $t_{k} \geqq \delta$, we have

$$
\beta_{k} \leqq \frac{\left|b_{k}-x_{k}\right|}{\left|x_{k}\right|}+1 \leqq \frac{\left|a_{k}-x_{k}\right|}{\delta\left|x_{k}\right|}+1 \leqq\left(\alpha_{k}+1\right) / \delta+1
$$

Hence $\alpha<\infty$ implies $\beta<\infty$. Similarly $\beta<\infty$ implies $\alpha<\infty$. If $\alpha=\beta=\infty$, we can write $\gamma_{k}=t_{k}+\varepsilon_{k}$ with $\varepsilon_{k} \rightarrow 0$, and we obtain the contradiction $\gamma \in[\delta, 1 / \delta]$. We divide the rest of the proof into three cases:

Case 1. $\alpha=\beta=0$. Then $t_{k} \rightarrow 1$. On the other hand, 5.21 implies

$$
\frac{\left|f_{k}^{\prime}\left(a_{k}\right)\right|}{\left|f_{k}^{\prime}\left(x_{k}\right)\right|} \rightarrow 0, \quad \frac{\left|f_{k}^{\prime}\left(b_{k}\right)\right|}{\left|f_{k}^{\prime}\left(x_{k}\right)\right|} \rightarrow 0,
$$

and hence $t_{k}^{\prime} \rightarrow 1$, which gives a contradiction.

Case 2. $\alpha=0,0<\beta<\infty$. We can write

where $\varepsilon_{k} \rightarrow 0$. As in Case 1 ,

$$
t_{k}=\frac{\left|x_{k}\right|}{\left|b_{k}-x_{k}\right|}+\varepsilon_{k}
$$

This implies

$$
\frac{\left|f_{k}^{\prime}\left(a_{k}\right)\right|}{\left|f_{k}^{\prime}\left(x_{k}\right)\right|} \rightarrow 0
$$

where $d_{k}^{\prime} \rightarrow 1$. Now

$$
t_{k}^{\prime}=\frac{d_{k}^{\prime}\left|f_{k}^{\prime}\left(x_{k}\right)\right|}{\left|f_{k}^{\prime}\left(b_{k}\right)-f_{k}^{\prime}\left(x_{k}\right)\right|}
$$

$$
\frac{\left|u\left(b_{k}\right)\right|}{\left|u\left(b_{k}\right)-u\left(x_{k}\right)\right|}=\frac{\left|x_{k}\right|}{\left|b_{k}-x_{k}\right|}=t_{k}-\varepsilon_{k} \leqq 1 / \delta-\varepsilon_{k} \leqq 1 / s_{k}
$$

for large $k$. Since $f_{k}$ is $s_{k}-\mathrm{QS}$ with $f_{k}(0)=0$, we obtain

Thus

$$
\frac{\left|f_{k}^{\prime}\left(x_{k}\right)\right|}{\left|f_{k}^{\prime}\left(b_{k}\right)-f_{k}^{\prime}\left(x_{k}\right)\right|}=\frac{\left|f_{k}\left(u\left(b_{k}\right)\right)\right|}{\left|f_{k}\left(u\left(b_{k}\right)\right)-f_{k}\left(u\left(x_{k}\right)\right)\right|} \leqq t_{k}-\varepsilon_{k}+s_{k} .
$$

$$
t_{k}+\delta \leqq t_{k}^{\prime} \leqq d_{k}^{\prime}\left(t_{k}-\varepsilon_{k}+s_{k}\right),
$$

which gives a contradiction as $k \rightarrow \infty$.

Case 3. $\beta=0,0<\alpha<\infty$. Now

$$
t_{k}=\frac{\left|a_{k}-x_{k}\right|}{\left|x_{k}\right|}+\varepsilon_{k}
$$


with $\left|\varepsilon_{k}\right| \leqq t_{k} \beta_{k} \rightarrow 0$. By 5.21,

$$
\frac{\left|f_{k}^{\prime}\left(b_{k}\right)\right|}{\left|f_{k}^{\prime}\left(x_{k}\right)\right|} \rightarrow 0 .
$$

For large $k$ we have $s_{k} \leqq \alpha_{k} \leqq 1 / s_{k}$, and then 5.21 implies

$$
\frac{1}{1 / \alpha_{k}+s_{k}} \leqq \frac{\left|f_{k}^{\prime}\left(a_{k}\right)\right|}{\left|f_{k}^{\prime}\left(x_{k}\right)\right|}=\alpha_{k}^{\prime} \leqq \alpha_{k}+s_{k},
$$

and hence $\alpha_{k}^{\prime} \rightarrow \alpha$. Now

where $d_{k}^{\prime} \rightarrow 1$.

$$
t_{k}^{\prime}=\frac{d_{k}^{\prime}\left|f_{k}^{\prime}\left(a_{k}\right)-f_{k}^{\prime}\left(x_{k}\right)\right|}{\left|f_{k}^{\prime}\left(x_{k}\right)\right|}
$$

We apply the $s_{k}$-quasisymmetry of $f_{k}$ to the triple $u\left(x_{k}\right), 0, u\left(a_{k}\right)$. Since

$$
\frac{\left|u\left(x_{k}\right)-u\left(a_{k}\right)\right|}{\left|u\left(a_{k}\right)\right|}=\frac{\left|a_{k}-x_{k}\right|}{\left|x_{k}\right|}=t_{k}-\varepsilon_{k} \leqq 1 / s_{k}
$$

for large $k$, we obtain

$$
\frac{\left|f_{k}^{\prime}\left(a_{k}\right)-f_{k}^{\prime}\left(x_{k}\right)\right|}{\left|f_{k}^{\prime}\left(x_{k}\right)\right|}=\frac{\left|f_{k}\left(u\left(x_{k}\right)\right)-f_{k}\left(u\left(a_{k}\right)\right)\right|}{\left|f_{k}\left(u\left(a_{k}\right)\right)\right|} \leqq t_{k}-\varepsilon_{k}+s_{k},
$$

which gives a contradiction as in Case 2 .

5.23. Theorem. Theorem 5.4 remains true if $R^{p}$ is replaced by $S^{p}$.

Proof. Let $s_{0}=s_{0}(n)$ and $s_{1}(s, n)$ be the numbers given by 5.4, and let $s_{0}^{*}=s_{0}^{*}(n)$ be the number given by 5.22. Suppose that $f: S \rightarrow R^{n}$ is an $s-$ QS map normalized as in 5.13. There is $s_{0}^{\prime}=s_{0}^{\prime}(n)>0$ such that if $s \leqq s_{0}^{\prime}$, we can choose a linear map $\alpha: R^{n} \rightarrow R^{n}$ as in the proof of 5.17. Now $\alpha$ is $L_{2}$-bilipschitz where $L_{2}=L_{2}(s, n) \rightarrow 1$ as $s \rightarrow 0$. It follows from 5.22 that if $s \leqq s_{0}^{*}$ and $s \leqq s_{0}^{\prime}$, then $\alpha f^{\prime}: T \rightarrow R^{n}$ is $s_{2}-\mathrm{QS}$ where $s_{2}=s_{2}(s, n) \rightarrow 0$ as $s \rightarrow 0$. Choose $s_{0}^{\prime \prime}=s_{0}^{\prime \prime}(n)>0$ such that $s_{0}^{\prime \prime} \leqq s_{0}^{*}, s_{0}^{\prime \prime} \leqq s^{0}$, and such that $s_{2}(s, n) \leqq s_{0}$ for $s \leqq s_{0}^{\prime \prime}$. If $s \leqq s_{0}^{\prime \prime}$, it follows from 5.4 that we can extend $\alpha f^{\prime}$ to an $s_{1}$-QS homeomorphism $g^{\prime \prime}: R^{n} \rightarrow R^{n}, s_{1}=s_{1}\left(s_{2}(s, n), n\right)$. By 5.11, we can choose $g^{\prime \prime}(0)=0$. By $2.6, g^{\prime \prime}$ is $K_{1}$-quasiconformal where $K_{1}=K_{1}(s, n) \rightarrow 1$ as $s \rightarrow 0$. Since $u$ is conformal, the map $g=u \alpha^{-1} g^{\prime \prime} u: R^{n} \rightarrow R^{n}$ is an $L_{2}^{2 n-2} K_{1}$ quasiconformal extension of $f$. By 2.6, $g$ is $s_{1}^{\prime}-\mathrm{QS}$ where $s_{1}^{\prime}=s_{1}^{\prime}(s, n) \rightarrow 0$ as $s \rightarrow 0$.

Added in proof. It is possible to simplify the proof of the QS case of 5.23 by using quasimöbius maps. These will be considered in a later paper of the second author.

\section{References}

[Ge $]_{1}$ Gehring, F. W.: Extension theorems for quasiconformal mappings in $n$-space. - Proceedings of International Congress of Mathematicians, Moscow, 1968, 313-318.

[Ge $\left.\mathrm{Ge}_{2}\right]$ Gehring, F. W.: Injectivity of local quasi-isometries. - Comment. Math. Helv. 57, 1982, $202-220$. 
[JK] Jerison, D. S., and C. E. KeniG: Hardy spaces, $A_{\infty}$, and singular integrals on chord-arc domains. - Math. Scand. 50, 1982, 221-247.

[La] LAtfullin, T. G., (Латфуллин Т. Г.): О геометрических условиях на образы прямой и окружности при квазиизометрии плоскости. - Материалы XVIII всесоюзной научной студенческой конференции, Новосибирск, 1980, 18-22.

$\left[\mathrm{Tu}_{1}\right]$ Tukia, P.: The planar Schönflies theorem for Lipschitz maps. - Ann. Acad. Sci. Fenn. Ser. A. I. Math. 5, 1980, 49-72.

$\left[\mathrm{Tu}_{2}\right]$ TukIA, P.: Extension of quasisymmetric and Lipschitz embeddings of the real line into the plane. - Ann. Acad. Sci. Fenn. Ser. A. I. Math. 6, 1981, 89-94.

[TV] TukiA, P., and J. VÄISÄLÄ: Quasisymmetric embeddings of metric spaces. - Ann. Acad. Sci. Fenn. Ser. A. I. Math. 5, 1980, 97-114.

[Vä] VÄIsÄL $\ddot{A}, J$.: Lectures on $n$-dimensional quasiconformal mappings. - Lecture Notes in Mathematics 229, Springer-Verlag, Berlin-Heidelberg-New York, 1971.

[Vä] ${ }_{2}$ V̈̈IsÄL ̈̈, J.: Quasi-symmetric embeddings in euclidean spaces. - Trans. Amer. Math. Soc. 264, 1981, 191-204.

University of Helsinki

Department of Mathematics

SF-00100 Helsinki 10

Finland

Received 1 March 1984 